\section{Eine gute Entscheidung}

Ich kann bereits nach einem Studienjahr sagen, dass sich mein Horizont aus fachlicher und persönlicher Sicht durch dieses Aufbaustudium erheblich erweitert hat. Was weiß der (die) Jurist(in) schon von Unternehmensstrategien? Oder was die Bekämpfung von Korruption mit Risikomanagement zu tun hat? Bei diesem Aufbaustudium lerne ich, wie wichtig es ist, über den Tellerrand zu blicken, um die Herausforderung „Compliance“ in allen Facetten zu bewältigen. Als Studierende kann ich mich mit Alumni - und damit weiteren Expert(inn)en aus allen Branchen auf den Gebieten - austauschen. Inzwischen zählt die School GRC rund 100 Absolvent(inn)en. Kontakte, Fachwissen und Titel runden diese zwei Jahre ab, so dass ich dieses Aufbaustudium nur empfehlen kann.

\title{
Veranstaltungsreihe „Alternative Berufsbilder im Recht“
}

Jungen Juristinnen und Berufsanfängerinnen fällt es oftmals schwer, die richtige Entscheidung im Hinblick auf ihren späteren Berufsweg zu treffen. Die wenigsten wissen bereits im Studium, in welchem Bereich sie später tätig sein möchten. Häufig bietet erst das Referendariat eine erste Orientierung und selbst dann haben nicht wenige junge Juristinnen Schwierigkeiten, sich für einen bestimmten Bereich zu entscheiden, wobei nicht zuletzt Unkenntnis über die Möglichkeiten juristischer Tätigkeit eine Rolle spielt.

Der djb veranstaltet daher in regelmäßigen $A b$ ständen Veranstaltungen für Student(inn)en, Referendar(inn)e(n) und Berufsanfänger(innen) im Hinblick auf die anstehende oder spätere Berufswahl. Diese finden unter dem Motto Alternative Berufsbilder an den Berliner Universitäten statt und wenden sich in erster Linie an diejenigen, die bei der Orientierung ihrer beruflichen Tätigkeit Unterstützung möchten. Der djb bemüht sich dabei, Möglichkeiten juristischer Tätigkeit abseits der traditionellen Berufe - als Anwältin, Staatsanwältin oder Richterin - oder aber ungewöhnliche Tätigkeitsfelder gerade in diesen Bereichen aufzuzeigen. Hierzu werden jedes Mal mehrere Referentinnen eingeladen, die über ihren Werdegang sowie ihre Tätigkeit berichten und im Anschluss daran an der Diskussion teilnehmen. Bisher fand in Berlin eine Reihe Veranstaltungen zu den Alternativen Berufsbildern im Recht statt, unter anderen zu folgenden Themen:
- Berufsbilder Öffentliches Recht: Erfahrungen erfolgreicher Juristinnen

- Berufsbild Anwältin: Erfahrungen erfolgreicher Juristinnen

- Berufsbild Strafrecht: Erfahrungen erfolgreicher Juristinnen

- Was ist, wenn ich nicht Richterin oder Anwältin werden will?

- Juristinnen in der Politik

- Juristinnen in der Wirtschaft

Dabei ergeben sich immer äußerst interessante Berichte der Referentinnen, bereichert durch eine allgemeine Diskussion im Anschluss, bei der den Zuhörer(inne)n die Möglichkeit gegeben wird, Fragen zu stellen und sich den einen oder anderen Tipp geben zu lassen. Die Veranstaltungsreihe soll auch weiterhin regelmäßig stattfinden. Soweit in einigen Landes- oder Regionalverbänden noch keine Veranstaltungen dieser Art organisiert worden sein sollten, stehen die jungen Juristinnen des Berliner Landesverbandes wie auch die Vertreterinnen der jungen Juristinnen im Bundesvorstand für Fragen und Tipps zur Verfügung. Ein entsprechender Leitfaden ist vorhanden und wird bei Anfrage an Katharina König aus dem Bundesvorstand (kathi16o1@web.de) gerne zugesandt. 